\title{
Effect of Mechanical Ventilation on Intestinal Permeability in Preterm Infants: A Retrospective Cohort Study
}

\author{
Martine F. Krüse-Ruijter ${ }^{1}$, Frans B. Plötz ${ }^{2}$, Jos W.R. Twisk ${ }^{3}$, Carin M. Bunkers ${ }^{4}$, \\ Willem P.F. Fetter ${ }^{1,4}$ and Ruurd M. van Elburg ${ }^{*}, 1,4$ \\ ${ }^{I}$ Department of Neonatology, ${ }^{2}$ Pediatric Intensive Care and the ${ }^{3}$ Institute of Research in Extramural Medicine, $V U$ \\ University Medical Center, Amsterdam, The Netherlands \\ ${ }^{4}$ Department of Neonatology, Isala Clinics location Sophia, Zwolle, The Netherlands
}

\begin{abstract}
Mechanical ventilation (MV) and ventilator strategies can induce or aggravate lung injury and may contribute to the development of distant organ failure, including the gastrointestinal tract. A retrospective cohort study was performed among 61 preterm infants, with a gestational age of 25-36 weeks, admitted at a neonatal intensive care unit. Intestinal permeability was measured by the sugar absorption test (SAT). Mechanically ventilated preterm infants were compared to not mechanically ventilated preterm infants. To analyze the effect of parameters of MV on intestinal permeability, we calculated the oxygenation index (OI). Intestinal permeability was not different in ventilated and not ventilated preterm infants within $48 \mathrm{hr}$ after birth. Although OI was $<10$ in most of the infants, OI was positively correlated with the SAT, suggesting that the degree of MV was correlated with intestinal permeability.
\end{abstract}

Keywords: Preterm infants, oxygenation index, mechanical ventilation, intestinal permeability, lactulose/mannitol ratio, sugar absorption test.

\section{INTRODUCTION}

Mechanical ventilation (MV) and ventilator strategies can induce or aggravate lung injury and may also contribute to the development of distant organ failure, including the gastrointestinal tract [1]. The barrier function of the gastrointestinal tract is amongst others reflected by intestinal permeability of macromolecules. Increased intestinal permeability can have negative effects, such as increased uptake of infectious agents leading to the development of infection, inflammation, and systemic hypersensitivity [2]. The mechanisms by which MV exert the effects on distant organs remain poorly understood. One possible effect of MV on the gastrointestinal tract is to increase the intestinal permeability, thereby promoting the translocation of bacteria or their products into the circulation. Various pro-inflammatory mediators, like tumour necrosis factor-alpha (TNF- $\alpha$ ), nuclear (transcription) factor (NF-אB) and interleukin-6 (IL-6), that have been shown to increase during MV, are known to influence the tight junctions in the gastrointestinal tract [3-6]. This suggestion is supported by an experimental study of Guery et al., who observed that high tidal volume ventilation resulted in an increase in lung oedema and gut permeability, which could be reversed by the administration of TNF neutralizing antibodies [7]. These observations suggest that the degree of MV is related to gut permeability. In our study, we use oxygenation index (OI) as a parameter of the degree of $\mathrm{MV}$. OI reflects the ventilatory response, incorporating mean

*Address correspondence to this author at the Department of Neonatology, VU University Medical Center, P.O. Box 7057, 1007 MB Amsterdam, The Netherlands; Tel: +31204442413; Fax: +31204443045;

E-mail: rm.vanelburg@vumc.nl airway pressure and oxygen requirement, and is considered an appropriate parameter to reflect pulmonary status in ventilated patients [8]. With high ventilator settings, the probability of ventilator-associated lung injury increases. High OI results from higher ventilator settings. Recently, Bayrakci et $a l$. showed that a high OI was a significant predictor for the development of chronic lung disease [9]. Previously, we showed that intestinal permeability is not related to gestational age or birth weight in preterm infants (25-36 weeks gestation) [10]. Furthermore, a sharp decline in intestinal permeability was found in the first week of life. Although we found no difference between so called "critically ill" infants, defined as infants on a ventilator, suffering birth asphyxia or small for gestational age, and healthy preterm infants, we did not investigate the role of MV or parameters of MV at that time. To our knowledge, the effect of MV on intestinal permeability in preterm infants has not been studied before.

Aims of this study were to compare intestinal permeability, as measured by the sugar absorption test (SAT), in preterm infants who were mechanically ventilated within 4 hours after birth and in preterm infants who were not ventilated after birth. In addition, to determine the correlation between intestinal permeability and ventilation strategies reflected by the oxygenation index in the ventilated preterm infants.

\section{MATERIALS AND METHODOLOGY}

Patients: A retrospective analysis of the patients, who had been included in a previously published prospective study at the neonatal intensive care unit of the Isala Clinics Zwolle was performed [10]. The hospital medical ethics committee of the Isala Clinics Zwolle approved the study. 
Written informed consent was obtained from the parents of all included infants.

In the present study only preterm infants, defined as a gestational age $<36$ weeks, were included. The preterm infants were divided in two groups. A control group consisted of preterm infants without RDS who were not ventilated at any time before the SAT was performed, and preterm infants with RDS who were intubated and mechanically ventilated within 4 hours after birth (MV group) and were still ventilated at the time of the SAT. Study infants received MV, either synchronized mandatory and/or positive pressure ventilation (SIMV and SIPPV respectively) in the first week of life. Infants with high frequency oscillatory ventilation or with necrotising enterocolitis were excluded. Perinatal data including maternal antibiotic use, apgar scores, gestational age, birth weight, the feeding regime, incidence of sepsis and necrotising enterocolitis, and mortality were retrieved from the medical records.

Methods: Intestinal permeability was measured by the SAT [10]. Briefly, 6-hour urinary excretion of lactulose and mannitol was measured by gas chromatography after instillation by nasogastric tube of $2 \mathrm{ml} / \mathrm{kg}$ of the sterile test solution containing $250 \mathrm{mg}$ lactulose and $100 \mathrm{mg}$ mannitol per $5 \mathrm{ml}$ water. Fasting before the test was not necessary. As a preservative, $0.1 \mathrm{ml}$ chlorohexidine digluconate $20 \%$ was added to the urine. Urine volume was measured and samples stored at $20^{\circ} \mathrm{C}$. Lactulose $(\mathrm{L})$ and mannitol $(\mathrm{M})(\mathrm{mmol} / \mathrm{mol}$ creatinine) were measured by gas chromatography and the L/M ratio was calculated. Between 24-48 hours after birth, the SAT was performed and ventilator settings were retrieved: mean airway pressure (MAP), positive inspiratory peak pressure (PIP), positive end-expiratory pressure (PEEP), inspiration and expiration time, flow, and inspiratory oxygen fraction $\left(\mathrm{FiO}_{2}\right)$. In addition, blood gas analysis values $\left(\mathrm{pH}, \mathrm{pCO}_{2}, \mathrm{HCO}_{3}\right.$, base excess and $\mathrm{pO}_{2}$ ) were retrieved. To analyze the effect of parameters of MV on intestinal permeability, we calculated the OI (if arterial blood gas samples were available) and correlated this parameter with the SAT. OI was calculated on the basis of the ventilator settings prior to the SAT. The following equation was used:

$\mathrm{OI}=\mathrm{FiO}_{2} * \mathrm{MAP} / \mathrm{PaO}_{2}$

Statistical analysis: All data are expressed as mean (SD) unless indicated otherwise. Data were analysed with SPSS 12.0 (SPSS Inc, Chicago, Illinois, USA), using the nonparametric Mann Whitney $U$ test, for comparing intestinal permeability of the groups and linear regression analysis for the effect of OI on intestinal permeability. One-way ANOVA with posthoc analysis and Bonferroni correction were used to compare the group characteristics. A p value $<$ 0.05 was considered statistically significant.

\section{RESULTS}

In total, 61 infants were included, of whom 21 were mechanically ventilated (Table 1). The MV group differed from the controls by a lower gestational age (GA) and lower Apgar score at $5 \mathrm{~min}$. There was no significant difference in baseline characteristics of the studied infants in sex, birth weight, umbilical cord $\mathrm{pH}$, sepsis or infection, maternal use of antibiotics or maternal dexamethason. All mechanically ventilated infants were ventilated primarily for neonatal lung diseases, of which respiratory distress syndrome (RDS) accounted for $86 \%$ and wet lung (also known as transient tachypnea of the newborn caused by incomplete removal of liquid from the lungs in the newborn at birth with symptoms such as grunting, retractions, and sometimes cyanosis, symptoms which usually resolves within 3 days after birth) for $5 \%$. At the time of SAT, the ventilator setting in the $\mathrm{MV}$ group were PIP $18 \pm 4 \mathrm{~cm} \mathrm{H}_{2} 0$, PEEP $4 \pm 1 \mathrm{~cm} \mathrm{H}_{2} 0$ and $\mathrm{FiO}_{2}$ $0.40 \pm 0.15$. In the control group, none of the control infants received supplemental oxygen.

Sugar absorption test (SAT): The SAT was performed at a mean (SD) postnatal age of $33.1 \pm 7.2$ and $33.6 \pm 6.1$ hours in the MV and control group, respectively. The median L/M ratio was not different in the MV and control group ( $\mathrm{p}=0.37$ ), also after correction for gestational age and for Apgar score at $5 \mathrm{~min}$ (Table $\mathbf{1}$ ).

Table 1. Baseline Characteristics of Patient Groups

\begin{tabular}{|c|c|c|c|}
\hline & Control Group N=40 & MV Group N=21 & P \\
\hline \hline GA (wks) & $31.6 \pm 2.4$ & $29.1 \pm 2.4$ & $<0.05$ \\
\hline BW (g) & $1452 \pm 484$ & $1359 \pm 475$ & NS \\
\hline AS 5 min & $8.7 \pm 1.1$ & $7.0 \pm 1.8$ & $<0.05$ \\
\hline Umbilical cord pH & $7.26 \pm 0.07$ & $7.24 \pm 0.18$ & NS \\
\hline SAT (hrs) & $33.6 \pm 6.1$ & $33.1 \pm 7.2$ & NS \\
\hline Sex (\% male) & 55 & 67 & NS \\
\hline RDS (\%) & 0 & 86 & $<0.05$ \\
\hline Wet lung (\%) & 28 & 5 & $<0.05$ \\
\hline Sepsis (\%) & 0 & 0 & NS \\
\hline Mat Dex (\%) & 78 & 67 & NS \\
\hline Maternal AB (\%) & 23 & 38 & NS \\
\hline Infection at SAT (\%) & 3 & 0 & NS \\
\hline L/M ratio* & 0.36 & 0.24 & NS \\
\hline
\end{tabular}

All data are expressed as mean +/-SD, except for L/M ratio* expressed as median (range). Abbreviations: GA gestational age, BW birth weight, AS Apgar score, SAT sugar absorption test, RDS respiratory distress syndrome, Mat Dex Maternal Dexamethason, $\mathrm{AB}$ antibiotics.

Because most infants were fed a mixture of mother's milk and preterm formula in the first days of life, we were unable to determine the influence of the type of enteral feeding on intestinal permeability.

The correlation between intestinal permeability (L/M ratio) and the OI is shown in Fig. (1). A significant correlation between OI and L/M ratio was found (after correction for gestational age and Apgar score at $5 \mathrm{~min}$, standardized coefficient Beta 0.57, $\mathrm{p}<0.02$ ) (Fig. 1).

OI at the time of SAT was $5.9 \pm 4.9$. Additional analysis revealed that the positive correlation between OI and L/M ratio was mainly due to permeability of lactulose. Permeability of lactulose differed from permeability of mannitol by a consistent rise in response to MV (Beta 0.59, p $<0.02$ and Beta $0.27, \mathrm{p}=0,31$, respectively). 


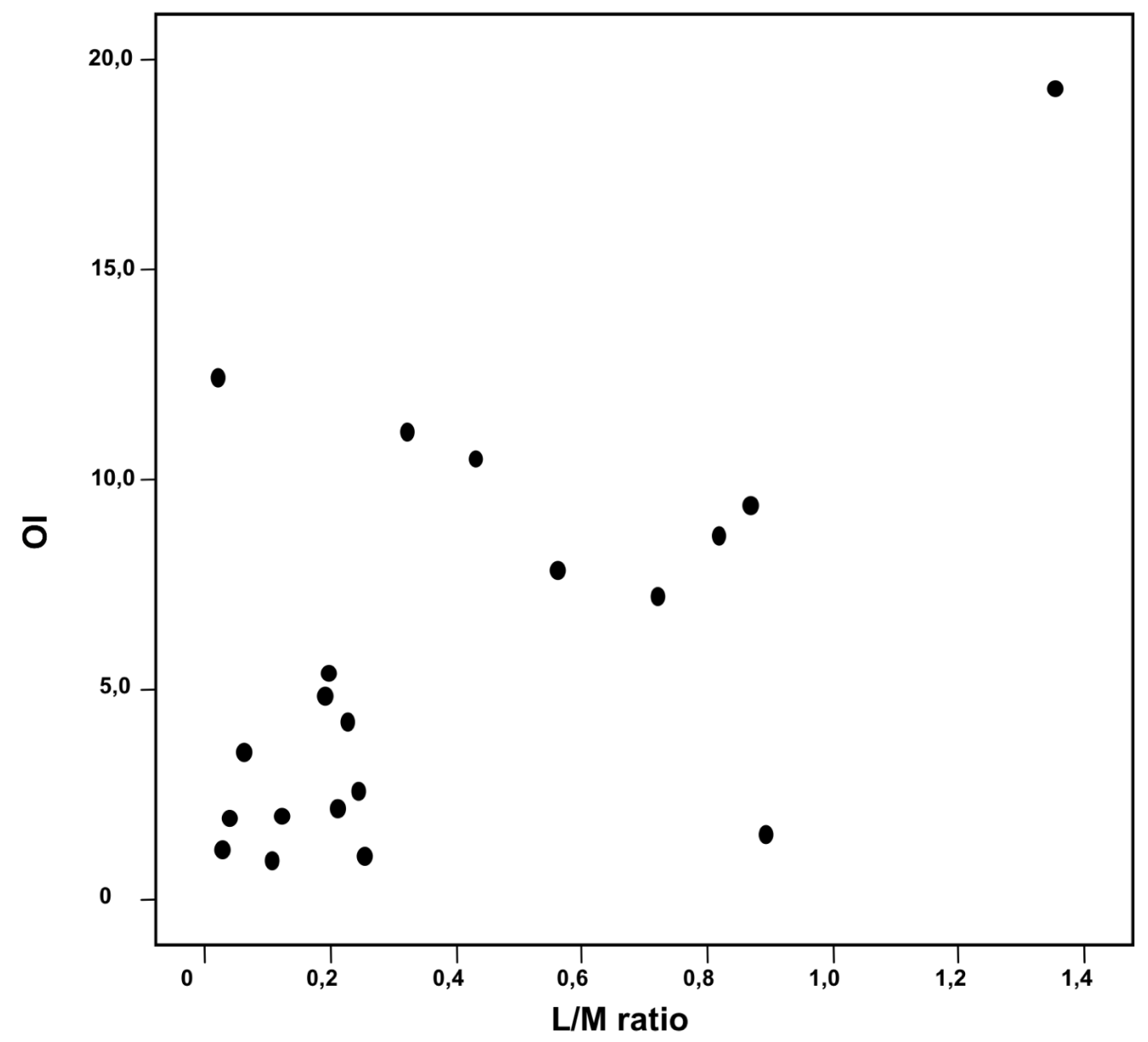

Fig. (1). Correlation between OI and L/M ratio at the SAT.

\section{DISCUSSION}

We found a positive correlation between the OI and L/M ratio, indicating that the degree of $\mathrm{MV}$ correlated with intestinal permeability. We could not contribute this correlation to differences in co-morbidities between the groups. However, in contrast to our expectations, MV did not increase the intestinal permeability in preterm infants. The L/M ratios were comparable among the groups.

In preterm infants, OI reflects the severity of the RDS [8]. Bayrakci et al. suggest that it is better to consider ECMO than conventional ventilation modalities to reduce the risk of developing chronic lung disease by high OI [9]. The OI levels in our NICU population are lower than the OI levels in the PICU population of Bayrakci [9], but better reflect the OI levels in a general NICU without ECMO facilities. Despite this fact, we did find a strong correlation with intestinal permeability. If we had included infants with more severe lung disease, and consequently with higher OI levels, the effect of OI and mechanically ventilation itself on intestinal permeability may have been more pronounced.

The mechanism by which MV may exert its effect on the gastrointestinal barrier remains unanswered. In this study, first, the positive correlation between the $\mathrm{OI}$ and the L/M ratio was mainly due to the permeability of lactulose. Lactulose is suggested to pass the small bowel mainly by the tight junctions [11]. Recently, it has become clear that factors, like oxi- dative stress, tumour necrosis factor-alpha (TNF- $\alpha$ and interleukin-6 (IL-6), were found to influence these tight junctions [3-6]. Second, it is known that MV induces a pulmonary inflammatory reaction, which depends on the degree of MV [12]. These lung-born inflammatory mediators, like TNF- $\alpha$ and IL-6, spill over in the systemic circulation thereby causing a systemic inflammatory response syndrome and may thus possible affect the gastrointestinal barrier $[1,13,14]$. Third, the pathophysiology of RDS also includes pulmonary and systemic inflammatory reaction [15]. Yulug et al. recently showed that one-lung ventilation causes significant tissue damage in the ileum of rats, with an increase in myeloperoxidase (MDA) and malondialdehyde (MPO) concentration, and histopathological changes of the ileum [16]. MDA is a marker of oxidative stress-mediated lipid peroxidation. Lipid peroxidation is believed to be an important cause of destruction and damage to cell membranes, leading to changes in membrane fluidity and permeability. MPO plays a role in oxidant production by neutrophils. Neutrophils are a potential source of oxygen free radicals, considered to be important in tissue damage and play a role in effects of oxidative stress. The increase in MDA and MPO activity suggests oxidative stress as a cause of the ileum tissue damage. Future studies should address these issues.

The results of our study are potentially clinically relevant despite the limitations of our study, i.e. retrospective design of the study, which may lead to incomplete data and does not 
allow a power analysis, and the absence of data on inflammatory mediators or markers for oxidative stress. Furthermore, during the study period all infants were ventilated in a pressure controlled mode, but data on tidal volumes were not routinely recorded. Correlation of tidal volumes with intestinal permeability would have been interesting in the current concept that ventilator-induced lung injury is primarily caused by administration of high tidal volumes [1,17]. It is known that the intestinal permeability is increased in critically ill patients $[18,19]$. Also the development of multiple organ dysfunction is associated with an increased intestinal permeability. Since most critically ill patients require mechanical ventilation, it is important to confirm our preliminary findings that the degree of MV may be an independent factor to influence intestinal permeability.

\section{CONCLUSION}

Intestinal permeability was not different in ventilated and not ventilated preterm infants within $48 \mathrm{hr}$ after birth. However, the OI was positively correlated with the SAT, suggesting that the degree of MV was correlated with intestinal permeability. Further studies are necessary to confirm our findings.

\section{ABBREVIATIONS}

\begin{tabular}{|c|c|}
\hline MV & $=$ Mechanical ventilation \\
\hline SAT & $=$ Sugar absorption test \\
\hline OI & $=$ Oxygenation index \\
\hline TNF- $\alpha$ & $=$ Tumour necrosis factor- $\alpha$ \\
\hline$N F-\kappa B$ & $=$ Nuclear (transcription) factor $-\kappa \mathrm{B}$ \\
\hline IL-6 & $=$ Interleukin- 6 \\
\hline SIMV & $\begin{aligned} &= \text { Synchronized intermittent mandatory } \\
& \text { ventilation }\end{aligned}$ \\
\hline SIPPV & $\begin{aligned}= & \text { Synchronized intermittent positive pressure } \\
& \text { ventilation }\end{aligned}$ \\
\hline $\mathrm{L}$ & $=$ Lactulose \\
\hline M & $=$ Mannitol \\
\hline $\mathrm{L} / \mathrm{M}$ ratio & $=$ Lactulose/mannitol ratio \\
\hline MAP & $=$ Mean airway pressure \\
\hline PIP & $=$ Positive inspiratory peak pressure \\
\hline PEEP & $=$ Positive end-expiratory pressure \\
\hline $\mathrm{FiO}_{2}$ & $=$ Inspiratory oxygen fraction \\
\hline GA & $=$ Gestational age \\
\hline RDS & $=$ Respiratory distress syndrome \\
\hline NICU & $=$ Neonatal intensive care unit \\
\hline PICU & $=$ Pediatric intensive care unit \\
\hline MDA & $=$ Myeloperoxidase \\
\hline MPO & $=$ Malondialdehyde \\
\hline
\end{tabular}

\section{REFERENCES}

[1] Plotz FB, Slutsky AS, van Vught AJ, Heijnen CJ. Ventilator induced lung injury and multiple system organ failure: a critical review of facts and hypotheses. Intensive Care Med 2004; 30: 186572.

[2] Insoft RM, Sanderson IR, Walker WA. Development of immune function in the intestine and its role in neonatal diseases. Pediatr Clin North Am 1996; 43: 551-71.

[3] Varella Morandi Junqueira-Franco M, Ernesto Troncon L, Garcia Chiarello P, do Rosario Del Lama Unamuno M, Afonso Jordao A, Vannucchi H. Intestinal permeability and oxidative stress in patients with alcoholic pellagra. Clin Nutr 2006; 25: 977-83.

[4] Spindler-Vesel A, Wraber B, Vovk I, Kompan L. Intestinal permeability and cytokine inflammatory response in multiply injured patients. J Interferon Cytokine Res 2006; 26: 771-76.

[5] Ye D, Ma I, Ma TY. Molecular mechanism of tumor necrosis factor-alpha modulation of intestinal epithelial tight junction barrier. Am J Physiol Gastrointest Liver Physiol 2006; 290: G496-G504.

[6] Fink MP. Intestinal epithelial hyperpermeability: update on the pathogenesis of gut mucosal barrier dysfunction in critical illness. Curr Opin Crit Care 2003; 9: 143-151.

[7] Guery B, Welsh DA, Viget NB, et al. Ventilation-induced lung injury is associated with an increase in gut permeability. Shock 2003; 9: 559-63.

[8] Lam BCC, Ng YK, Wong KY. Randomized trial comparing two natural surfactants (Survanta vs Bles) for treatment of neonatal respiratory distress syndrome. Pediatr Pulmonol 2005; 39: 64-69.

[9] Bayrakci B, Josephson C, Fackler J. Oxygenation index for extracorporeal membrane oxygenation: is there predictive significance? J Artif Organs 2007; 10: 6-9.

[10] Van Elburg RM, Fetter WPF, Bunkers CM, Heymans HS. Intestinal permeability in relation to birth weight and gestational and postnatal age. Arch Dis Child Fetal Neonatal Ed 2003; 88: F52-F55.

[11] Samonte VA, Goto M, Ravindranath TM, et al. Exacerbation of intestinal permeability in rats after a two-hit injury: burn and Enterococcus faecalis infection. Crit Care Med 2004; 32: 2267-73.

[12] Tremblay L, Valenza F, Ribeiro SP, Li J, Slutsky AS. Injurious ventilatory strategies increase cytokines and c-fos m-RNA expression in an isolated rat lung model. J Clin Invest 1997; 99: 944-52.

[13] Ranieri VM, Suter PM, Tortorella C, et al. Effect of mechanical ventilation on inflammatory mediators in patients with acute respiratory distress syndrome: a randomized controlled trial. JAMA 1999; 282: 54-61.

[14] Parsons PE, Eisner MD, Thompson BT, et al. NHLBI Acute Respiratory Distress Syndrome Clinical Trials Network: Lower tidal volume ventilation and plasma cytokine markers of inflammation in patients with acute lung injury. Crit Care Med 2005; 33: 1-6.

[15] Brus F, Van Oeveren W, Okken A, Bambang Oetomo S. Number and activation of circulating polymorphonuclear leukocytes and platelets are associated with neonatal respiratory distress syndrome severity. Pediatrics 1997; 99: 672-80.

[16] Yulug E, Tekinbas C, Ulusoy H, et al. The effects of oxidative stress on the liver and ileum in rats caused by one-lung ventilation. J Surg Res 2007; 139: 253-60.

[17] The Acute Respiratory Distress Syndrome Network. Ventilation with lower tidal volumes as compared with traditional tidal volumes for acute lung injury and the acute respiratory distress syndrome. N Engl J Med 2000; 342: 1301-8

[18] Harris CE, Griffiths RD, Freestone N, Billington D, Atherton ST, Macmillan RR. Intestinal permeability in the critical ill. Intensive Care Med 1992; 18: 38-41.

[19] Doig CJ, Sutherland LR, Sandham JD, Fick GH, Verhoef M, Meddings JB. Increased intestinal permeability is associated with the development of multiple organ dysfunction syndrome in critically ill ICU patients. Am J Respir Crit Care Med 1998; 158: 444-51. 